\title{
Arbor
}

\section{El hombre en el espacio cósmico}

\author{
Koptiev Yuri Nikoláyevich
}

Arbor CLXXI, 673 (Enero), 129-144 pp.

"Los vuelos al espacio cósmico no se pueden detener. Esta no es una tarea de un sólo hombre cualquiera ni siquiera de un grupo de gente. Es un proceso histórico al que la humanidad llegó en su desarrollo normalmente».

Yuri Gagarin (Primer cosmonauta del planeta Tierra)

En el artículo se examinan las cuestiones relacionadas con el vuelo del hombre al espacio cósmico. Al principio se enumeran mitos y obras de ficción de los siglos pasados en los que se exponían diferentes ideas y métodos de vuelo del hombre al espacio, entre éstos, los que proponian los científicos españoles. Más adelante, se mencionan los principales logros de la técnica espacial de cohetes de Rusia y, en primer lugar, del primer vuelo espacial del mundo de Yuri Alekséyevich Gagarin el 12 de abril de 1961. Se pone especial atención en la resolución de uno de los problemas más importantes: la creación del cohete portador que garantizó la puesta en órbita terrestre de la nave espacial de Gagarin «Vostok».

En el artículo, la atención principal se presta a la estación «Mir». Se describe brevemente la prehistoria de su creación, se exponen los resultados de las investigaciones realizadas y se presentan los informes globales de los resultados de su vuelo de 15 años de duración.

La idea del vuelo del hombre al espacio cósmico no surgió en el siglo XX ni fue en Rusia o en USA, aunque ésta fue puesta en práctica por la ex Unión Soviética en el año 1961.

$\mathrm{El}$ cosmos, increíblemente lejano y misteriosamente atrayente desde los tiempos inmemoriales, agitaba la imaginación de las personas. El hombre siempre trataba de comprender las leyes de la creación del mun-

* Traducción al español: Carmen Alonso Cecilia 
do y conocerse a sí mismo, encontrar a seres semejantes en los vastos espacios del Universo.

El sueño del hombre de introducirse en el cosmos y su ambición de llegar a las estrellas nacieron hace miles de años y encontraron su reflejo en el folklore y la literatura de muchos pueblos. Los relatos sobre estos hechos se encuentran en la épica de los asirios y babilonios, en las antiguas leyendas chinas, iraníes y griegas, en los antiguos poemas indios y otras creaciones del pasado lejano.

El nivel de conocimientos de aquellos tiempos se reflejaba en los métodos de vuelo al espacio de los héroes de los poemas épicos. Al principio, el vuelo imaginario se realizaba sobre pájaros. Por ejemplo, se conocen los mitos del vuelo del rey babilónico de Etána sobre un águila hace 3200 años antes de nuestra era, del vuelo de Alejandro de Macedonia sobre grifones. Es inmortal el mito griego del vuelo de Ícaro hacia el Sol mediante unas alas de plumas de ave unidas a su cuerpo con cera. Sobre cisnes amaestrados realizó su vuelo el héroe de la novela de F.Godwin «El hombre en la Luna», editada en 1638. Además de servirse de pájaros, caballos, alas artificiales, huracanes y erupciones de volcanes para volar hacia las lejanías celestes, la fantasía de la gente utilizó las fuerzas místicas con mucha frecuencia.

Hace más de 300 años aparecieron las novelas de ficción, en las que por primera vez se describían los métodos de vuelo mecánico. Entre éstos, en la obra del escritor francés Cyrano de Bergerac (1649) por primera vez se menciona un vuelo con ayuda de cohetes a pólvora disparados sucesivamente.

El poeta inglés Lord (George) Byron creía que era posible alcanzar la Luna con ayuda de una máquina a vapor, y describió este método en su obra «Don Juan» (1818-1824). En sus conocidas novelas «De la Tierra a la Luna» (1865) y «Alrededor de la Luna» (1870), Julio Verne envía a sus héroes a la Luna en una bala de cañón, provista de propulsores de cohetes para corregir su trayectoria. También en su novela «Los quinientos millones de la Begum» (1879) aparece la idea de creación de satélites artificiales de la Tierra con ayuda de cohetes disparados con un cañón.

El escritor ruso A. Bogdánov en su obra «Estrella roja» (1908) optó por «menos-materia» para un vuelo a Marte en combinación con un motor a reacción que emplea la energía de desintegración atómica de la materia.

También existen informes sobre el interés que se manifiesta en España hacia la técnica de cohetes. Así, entre los años 1537 y 1540, Luis Ortiz escribió un tratado, «Libro sobre artillería», que es un trabajo muy antiguo sobre esta temática redactado por un autor español. 


\section{El hombre en el espacio cósmico}

En 1592, salió el libro del sevillano Luis Collado denominado «Plática Manual de Artillería», en el que se describe el empleo de cohetes por los ejércitos del rey Carlos V.

En 1872, fue publicado el trabajo del profesor Federico Gómez, director de la escuela naval de Barcelona, donde se ilustraban las cuestiones de vuelo en aparatos más pesados que el aire.

Es posible que este mismo autor publicara, en 1876, el proyecto de un aparato volador en cohete destinado para el vuelo del hombre dentro de los límites de la atmósfera terrestre.

Se pueden dar muchos ejemplos de cómo, desde tiempos inmemoriales, los terrícolas han fantaseado sobre los vuelos al espacio. Pero la ciencia en desarrollo rechazó la fuerza de tracción viva para vuelos hacia las alturas, estableció una extensión limitada de la atmósfera, y por esta causa, la incapacidad de los medios aeronáuticos y de aviación para vuelos al espacio cósmico determinó la insuficiente efectividad del vapor como medio motriz para las naves espaciales y la imposibilidad del empleo de cañones para este fin.

Por fin, a principios del siglo XX, en Rusia, Constantín Ziolkovski (1875-1935) elaboró la teoría del vuelo en cohete, los principios básicos de la construcción de sistemas de cohetes espaciales y los primeros planes científicos para que el hombre alcance el cosmos.

El trabajo publicado por él en 1903, «Investigación de los Espacios universales con aparatos a reacción» y sus complementos posteriores, son una aportación fundamental al tesoro de la ciencia mundial.

Los estudios teóricos de Ziolkovski fueron confirmados más tarde, y se comple-taron con investigaciones científicas tanto en Rusia, como en el extranjero: en Francia, por Eno-Peltri, 1913, 1928, 1930-1935; en USA, por Robert Goddard, 1919, 1936; en Alemania, por German Obert, 1923,1929, y por otros científicos notables.

Ha transcurrido más de medio siglo desde el día de la publicación del trabajo clásico de Ziolkovski, antes de los acontecimientos memorables de la historia, cuando se rompieron las cadenas de la gravitación terrestre. El 4 de Octubre de 1957, día del lanzamiento del primer satélite artificial de la Tierra por la Unión Soviética, y el 12 de Abril de 1961, 108 minutos del primer vuelo de un hombre, de Yuri Gagarin al cosmos en la nave «Vostok»: estos dos acontecimientos abrieron la era espacial de la humanidad y entraron para siempre en la historia de la civilización mundial.

Es completamente normal el interés que existe hacia cómo sucedió esto y qué éxitos fueron posibles gracias a esto.

Los primeros lanzamientos cósmicos alteraron la mente de la humanidad. Las posibilidades alcanzadas por la técnica de cohetes espacial en 
el conocimiento y dominio del mundo que nos rodea, han obligado a considerar de nuevo todo el sistema de valores humanos-filosóficos, morales, políticos y económicos, ver de distinta manera la resolución de los problemas nacionales, entre estados y de toda la humanidad.

En los años transcurridos desde el día del lanzamiento del I SAT (satélite artificial terrestre), y después del vuelo de Yuri Gagarin, el progreso en la creación de los sistemas de cohetes espaciales y la introducción, relacionada con éste, en el espacio, iban sucediendo a ritmos rápidos.

Los vuelos de los primeros satélites artificiales de la Tierra, Sol, Luna, Venus y Marte, los éxitos de haber alcanzado por primera vez con aparatos automáticos las superficies de la Luna, Venus y Marte y aterrizado suavemente en estos cuerpos celestes; las fotografías de la parte inversa de la Luna y la transmisión a la Tierra de imágenes de la superficie lunar, el vuelo alrededor de la Luna y el regreso a la Tierra de la nave automática con animales, las muestras de rocas lunares traídas a la Tierra por un robot, la investigación de la superficie de la Luna por el lunojod automático, la transmisión del panorama de Venus a la Tierra, el vuelo cerca del núcleo del cometa Halley, los vuelos de los primeros cosmonautas - hombres y mujeres, sus primeras salidas al espacio abierto, la creación de las primeras estaciones orbitales pilotadas, de una nave de carga automática de abastecimiento, los numerosos vuelos de tripulaciones internacionales, los primeros traslados de los cosmonautas entre las estaciones orbitales «Saliut-7» y «Mir», la construcción de un sistema transportador cósmico "Energía» - «Burán» con regreso automático total de la nave lanzadera espacial a la Tierra al punto fijado y muchos otros logros de Rusia, son conocidos por todos en el mundo.

Ha quedado para la historia el siglo XX, lleno de numerosos descubrimientos científicos y tecnológicos. Y sin embargo, los acontecimientos del siglo pasado más brillantes, emocionalmente saturados y a su manera conocidos en el ámbito de los vuelos espaciales pilotados, fueron la primera introducción del hombre en el espacio cósmico y el vuelo más prolongado, de 15 años, de la estación orbital «Mir».

Las generaciones de los mayores recuerdan bien el Abril de 1961, cuando todo el mundo fue sorprendido por la hazaña audaz de Yuri Gagarin (figura 1). El vuelo de la nave «Vostok» fue sencillamente éxito del pensamiento científico y técnico.

Sobre este vuelo fueron escritos centenares de libros y artículos; por esta razón, quisiera señalar aquí uno de los problemas más complicados que resolvieron los investigadores de Rusia, y como se sabe, sobre los hombros de los primeros descubridores, recaen las pruebas más duras. 


\section{El hombre en el espacio cósmico}

Esta fue la creación del cohete portador que garantizó la llegada del satélite artificial pilotado a la órbita terrestre.

En la Unión Soviética, el desarrollo práctico de la industria de cohetes comenzó aún en los años 30 . En aquellos tiempos se habían realizado numerosas investigaciones teóricas y experimentales, pero éstas fueron interrumpidas por la segunda guerra mundial. Muy poco antes de su comienzo, se crearon los famosos proyectiles a reacción «Katiushas» que contribuyeron enormemente a ganar la guerra.

Un gran auge adquirieron los trabajos en el ámbito de la técnica de cohetes en los años de posguerra. A finales de los años 40, empezó la gran batalla técnica. Ésta no se llevó a cabo en los campos de batalla, sino en las oficinas de diseño y proyec-

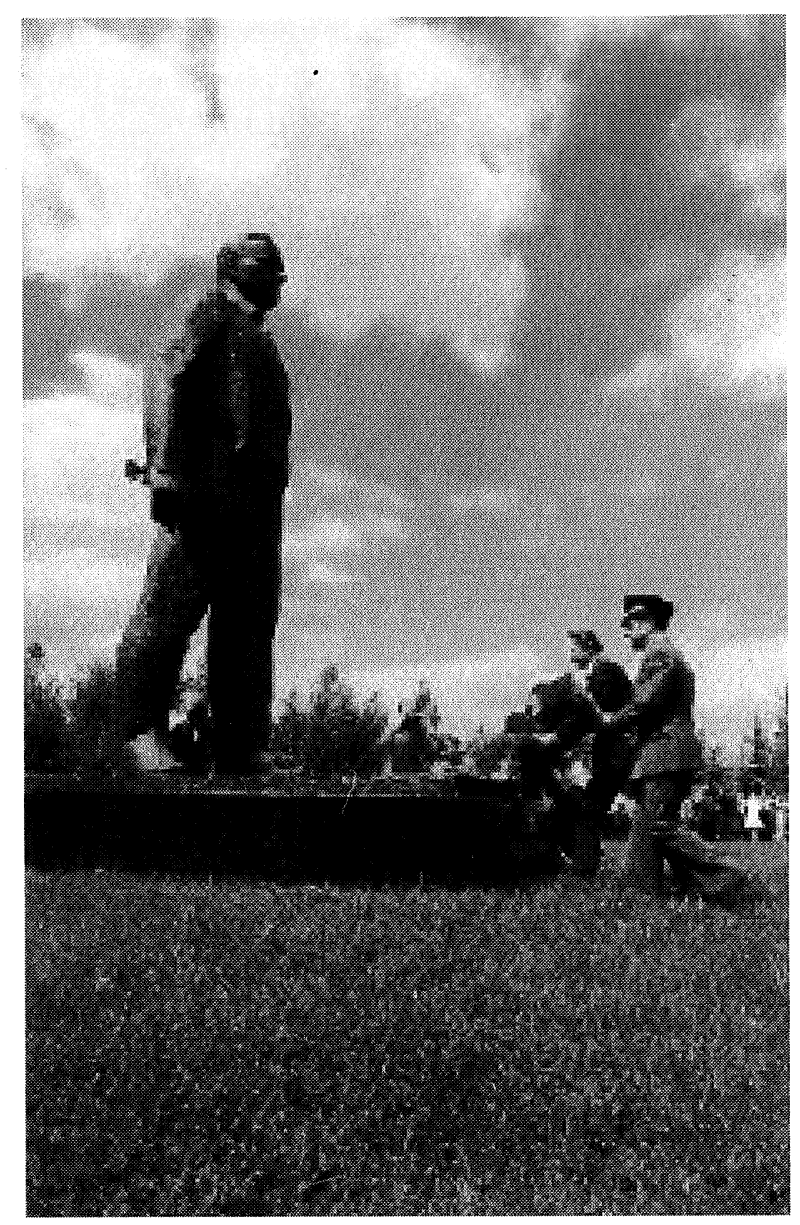

FIGURA 1

Monumento a Yuri Gagarin (1934-1968) en la Ciudad de las Estrellas tos, laboratorios, talleres de las fábricas, en los polígonos y en el silencio de los despachos de los científicos.

En la URSS y en USA se construían cohetes balísticos. El espíritu de competición, la rivalidad en la técnica y ciencia (como en el deporte) trae consigo, por regla, la obtención de resultados más altos y en plazos más cortos.

En diez años, desde 1946 a 1956, bajo la dirección del eminente diseñador Serguey Pávlovich Koroliov se construyeron varios tipos de cohetes balísticos de escalón único. Y en el año 1954, comenzó la elaboración del primer cohete balístico intercontinental. Sus creadores nece- 
sitaban elegir el esquema de composición de un cohete más seguro y óptimo en cuestiones energéticas; crear propulsores líquidos potentes, un sistema de mando del movimiento que asegurase las precisiones totalmente insólitas para aquellos tiempos; construir un complejo de despegue, elaborar la tecnología de preparación y pruebas de antes del despegue, así como la técnica de medición de las trayectorias. Este cohete de dos escalones fue construido en unos plazos extraordinariamente cortos, incluso según las medidas actuales de tiempo, y el 21 de agosto de 1957 tuvo lugar su exitoso lanzamiento.

Precisamente este cohete es la base de toda la familia de cohetes portadores, cuyas últimas modificaciones se emplean hasta nuestros días. Gracias a este cohete fue lanzado el primer satélite artificial de la Tierra el 4 de Octubre de 1957, y fue posible el vuelo pilotado de Yuri Gagarin en la nave «Vostok» el 12 de Abril de 1961.

El desarrollo posterior de los vuelos pilotados transcurría por el camino de una constante complicación, tomando en cuenta los resultados científicos y técnicos alcanzados y la aparición de nuevos problemas científicos, económicos y técnicos. Desde las primeras naves pilotadas y estaciones orbitales hasta los complejos multimodulares de «Mir» y la Estación cósmica internacional -este fue el camino recorrido por la cosmonáutica de Rusia-.

La estación orbital es el lugar de trabajo de los cosmonautas, también su hogar, donde ellos descansan después de su jornada laboral, y la cocina, donde se conservan las reservas de agua, alimentos y donde los cosmonautas comen; también es un complejo de saneamiento y de higiene. Los constructores de las estaciones han tropezado con numerosos problemas, el principal de los cuales era garantizar la seguridad de la tripulación en un vuelo prolongado. Los diseñadores tuvieron que determinar toda una serie de medidas de prevención. Si surgen defectos técnicos en la estación, la tripulación debe tener siempre la posibilidad de abandonarla y regresar a la Tierra en una nave de transporte que constantemente se encuentra junto al amarradero.

Antes del «Mir» fueron construidas las estaciones orbitales "Saliut», de primera generación, y 2 estaciones de segunda generación: «Saliut-6» y «Saliut-7». Las últimas dos estaciones se distinguían de las anteriores en muchas particularidades de construcción y en más amplias posibilidades para las investiga-ciones. Han aumentado su duración y el nivel de confort. Éstos ya tenían no un único sino dos nudos de empalme, garantizaban la posibilidad de salida al espacio abierto de dos cosmonautas al mismo tiempo, el repostado total de combustible del mecanismo motor unificado y muchos otros cambios. 


\section{El hombre en el espacio cósmico}

El defecto principal de las estaciones «Saliut» era su estructura compuesta de un monobloque. Este hecho limitaba la utilización de los aparatos científicos. Otro defecto era la ausencia de órganos ejecutivos electromecánicos, con lo que para orientarse eran necesarias considerables reservas de combustible y, consecuentemente, un gran flujo de carga «Tierra-bordo». En los «Saliut» no existía un sistema de comunicación a través del satélite-retransmisor, y la comunicación con las estaciones tenía grandes interrupciones. La experiencia obtenida en los «Saliut» y en las naves «Soyuz», relativo al funcionamiento de los sistemas de mando sobre la base de la técnica numérica, permitió pasar a los sistemas de mando modernos con la utilización del CNCB (complejo numérico computador de a bordo).

En estas condiciones pues comenzaron los trabajos de la estación «Mir». En 1976, la Sociedad de producción científica «Energía» (organización principal en la creación de naves y estaciones pilotadas) emitió unas proposiciones técnicas de creación de estaciones orbitales de larga duración. Y en agosto de 1978, fue elaborado un anteproyecto basado en la utilización de los sistemas de las estaciones «Saliut», pero complementariamente a los dos nudos de empalme axial se preveían cuatro laterales para el empalme de los módulos científicos. En febrero de 1979, salió la Disposición del gobierno sobre el desarrollo de los trabajos de construcción de estaciones de nueva generación; se determinó la cooperación de las organizaciones de elaboración y producción del bloque básico, de la maquinaria de a bordo y terrestre, en la que participaban más de 100 organizaciones de 20 ministerios y departamentos.

La estación orbital «Mir» se convirtió en la continuación lógica del programa en que el hombre se estableciera por etapas en el espacio cósmico terrestre (figura 2). Este programa integró toda la experiencia de sus predecesoras -las estaciones orbitales de larga duración de la serie «Saliut», y en particular, las dos últimas, «Saliut-6»y «Saliut-7»-.

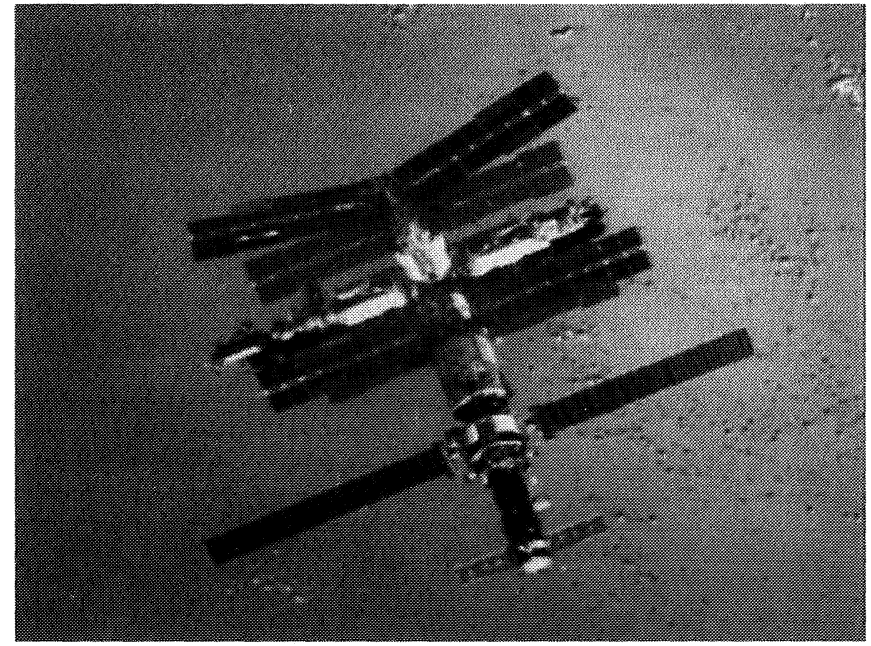

FIGURA 2

La estación espacial MIR (1986-2001) 
El primer elemento de la estación, su bloque básico, fue lanzado a la órbita el 20 de febrero de 1986. El lanzamiento se realizó con ayuda del cohete portador «Protón», que despegó desde el cosmódromo de Baykonur. La estación «Mir» fue proyectada de manera que en su estructura de sólo un bloque básico, ésta ya era apta para su explotación y podía garantizar las condiciones necesarias para el trabajo prolongado de los tripulantes a bordo. Pero este lanzamiento fue solo el comienzo de construcción única fuera de la Tierra -la creación de un complejo multiobjetivo de tipo modular pilotado que funciona constantemente en órbita-.

La construcción modular permitía hacer flexibles no sólo los programas de las investigaciones científicas, sino también los procesos de búsqueda de las soluciones de ingeniería y construcción de equipamiento complementario de la estación espacial. La estación «Mir» alcanzó su configuración total en 1996, cuando en su conjunto se incluyó el elementomódulo «Priroda» (naturaleza).

Las singularidades de la estación «Mir», que ventajosamente la distinguen de sus predecesoras, las estaciones «Saliut», además de sus módulos, eran tales soluciones técnicas y de proyectos como: su aptitud para las reparaciones, su adaptabilidad, utilización de la nueva base de elementos, aplicación del alto nivel de reserva, de sistema de orientación y estabilización en los giróscopos de fuerza de poco consumo y de duplicación funcional en la ejecución de los regímenes de trabajo más responsables. Todas estas singularidades fundamentales, cuya alta efectividad fue confirmada por su explotación de 15 años, llegaron a convertirse en principios clásicos peculiares al construir complejos orbitales de largo alcance.

La concepción de construcción modular de una estación y de sus sistemas, aseguró el desarrollo por etapas del complejo orbital, que excluía las consecuencias catastróficas al surgir situaciones peligrosas extraordinarias. El alto grado de viabilidad de la «Mir», gracias a su construcción por módulos, fue claramente demostrado el 25 de junio de 1997, cuando a consecuencia del choque con la nave de carga «Progress M-34» tuvo lugar la deshermetización del módulo «Spektr». Este módulo fue simplemente aislado de los demás compartimientos de la estación. Pero a pesar de todo, la parte de su equipamiento seguía utilizándose. Los cosmonautas realizaron los trabajos necesarios de conexión de sus baterías solares al sistema de abastecimiento de energía de la estación y el restablecimiento del funcionamiento del sistema de orientación de estas baterías hacia el Sol.

La posibilidad de realizar los trabajos de reparación y profilaxis operativas permite eliminar durante el vuelo los fallos más complejos, incluyendo los imprevistos, y liquidar las averías. El sistema previsto de abastecimiento material y técnico de la estación en la base de las naves de 
carga automáticas «Progress», demostró su adaptabilidad a los posibles cambios del programa de los trabajos en la estación y su alta efectividad.

La elaboración del programa de vuelo mediante la combinación de las expediciones principales que aseguran una constante permanencia de la tripulación a bordo con expediciones de permanencias más cortas, permite planificar con operatividad los programas de las investigaciones, dependiendo de la especificación de las prioridades de los problemas a solucionar y de la disposición de los aparatos. Los principios fundamentales presentaron las exigencias correspondientes también para la creación de los sistemas de a bordo de la estación «Mir», donde se utilizaron por principio las nuevas soluciones científico-técnicas. Esto, ante todo, se refiere al sistema de mando de la estación, en la que la utilización de nuevos enfoques de elaboración, así como las posibilidades de la técnica de cálculo, permitieron modernizar y sustituir, en caso de necesidad, la garantía programática del circuito radioeléctrico, realizar la complejidad de los medios de cálculo de módulos aislados y resolver los problemas de mando de orientación de la estación con ayuda de volantes-girodinas, lo cual redujo las necesidades de consumo de combustible enviado desde la Tierra.

Además, fueron creados equipos de empalme de varios tipos y modificaciones, un sistema automático de reempalme de los grandes módulos desde los nudos axiales a los laterales de empalme, dispositivos de despliegue y repliegue repetidos de las baterías solares. Se redujo considerablemente el flujo de carga desde la Tierra de materiales de consumo gracias a la creación de sistemas parcialmente cerrados y de los medios de habitabilidad.

El sistema de radiocomunicación por satélite aumentó considerablemente las posibilidades de interacción con la tripulación en órbita, prácticamente aseguró la transmisión de información continua sobre el estado de los sistemas de a bordo y los resultados de las investigaciones, lo cual por principio cambió el concepto de mando del vuelo.

En el transcurso del vuelo de la estación «Mir», en su superficie superior aparecieron cambios sustanciales de construcción. En el proceso de sus salidas, los cosmonautas montaron nuevas baterías solares, ensamblaron las estructuras de armaduras-postes (mástiles) de amarre. En la cima del más alto de ellos, de 14 metros «Co.17», colocaron un dispositivo motor de salida para el mando de la estación según su inclinación.

En el transcurso del vuelo, en el exterior de la estación se construyeron dos "grúas» cósmicas - puntales telescópicos de carga-, que se convirtieron en un medio insustituible para el transporte de cargas y de cosmonautas hacia el lugar de los trabajos durante el proceso de sus salidas al espacio abierto. 
Los traslados interorbitales desde la estación «Mir» a la estación «Saliut7» y de vuelta, han abierto por principio una nueva página en el dominio del espacio terrestre, permitiendo realizar en la práctica la explotación simultanea y complementaria recíproca de varios complejos cósmicos pilotados.

El sistema de mando teleoperador a distancia de las naves automáticas de transporte al empalmar con la estación aumentó considerablemente la seguridad de realización de las maniobras orbitales de aproximación y montaje.

Prácticamente en todas las direcciones de creación de una estación y sus sistemas de a bordo, se realizaron estudios en el plano científico-técnicos de vanguardia, se utilizaron nuevos materiales únicos, se asimilaron los procesos tecnológicos ultramodernos.

Durante el vuelo se obtuvieron resultados de valor excepcional, que contribuyeron por principio en el desarrollo de los conocimientos sobre el Universo y la materia, sobre los factores globales que influyen sobre nuestro planeta y el espacio terrestre, sobre el organismo del hombre y las formas de evolución de la vida en total.

Con instrumentos astrofísicos se obtuvieron espectros e imágenes radiológicas del centro de nuestra Galaxia. Por primera vez se registró la radiación radiológica de Supernova en la constelación de Zorra, se descubrieron fuentes radiológicas nuevas más alejadas de la Tierra y se registraron Candidatos antes desconocidos a «agujeros negros».

Los datos de los recursos naturales de la Tierra y seguimiento ecológico se utilizaron con operatividad para la renovación de mapas, control del terreno y de la situación ecológica.

Una considerable atención se prestaba a las observaciones de los procesos que suceden en la atmósfera superior y que juegan un papel ponderable en el balance ecológico de la Tierra y, posiblemente, determinantes del futuro de la humanidad.

En el ámbito de las investigaciones geofísicas se obtuvieron datos de las características espaciales, energéticas y temporal de los flujos de partículas de alta energía en el espacio cósmico terrestre, de los mecanismos de su generación, de la interrelación con los fenómenos y procesos heliofísicos en el espacio interplanetario. Continuó la formación del paquete de datos sobre los posibles precursores ionosféricos de terremotos, de elaboración de métodos de su monitoring con ayuda de complejos pilotados.

Sobre la base de las investigaciones de la física del líquido, transferencia de masa y calor y cristalización en condiciones de ingravidez, se han elaborado métodos de obtención de materiales-cristales nuevos y valiosos, que superan los análogos terrestres, de arseniuro de galio, telururo de cadmio y óxido de zinc.

A bordo de la estación "Mir» se desplegaron investigaciones intensivas de obtención y transformación de los biopreparados, con propiedades 


\section{El hombre en el espacio cósmico}

previamente determinadas, para su introducción en las empresas de industria microbiológica.

En la esfera de las investigaciones médico-biológicas se ha determinado una serie de importantes regularidades de adaptación del organismo del hombre a los factores del vuelo espacial. Se han resuelto los problemas médicos de garantía de la seguridad de los vuelos de duración de hasta un año y medio (1,5). Los resultados obtenidos se han implantado y se emplean con éxito en condiciones de superficie terrestre en el ámbito de las investigaciones clínico-fisiológicas, de la medicina extrema, ecología, control sanitario e higiénico y seguridad radioactiva.

Se consiguieron éxitos considerables también en la organización del funcionamiento de la industria de Rusia. La finalidad de resolver el problema principal permitía a cortos plazos superar los numerosos problemas particulares, integrar los esfuerzos de numerosos colectivos. Sobre la base del trabajo creativo conjunto formó y confirmó la capacidad de vida en condiciones extremas de déficit de medios financieros, la cooperación de muchos miles, que incluía las organizaciones prácticamente de todas las ramas de la ciencia, técnica e industria.

Y he aquí que llegó la hora del cese del vuelo de la estación «Mir». El 30 de diciembre de 2000, se aprobó la disposición del gobierno de Rusia «Sobre la conclusión del trabajo del complejo orbital pilotado «Mir». El gobierno aprobó las proposiciones de Rosaviakosmos (Avia-cosmos de Rusia) sobre el hundimiento civilizado de los fragmentos del complejo «Mir» en la región fijada del océano Pacífico en febrero-marzo de 2001.

El 24 de enero de 2001 despegó y el 27 de enero empalmó con la estación la nave de transporte «Progress-M1-5» con reserva precisa de combustible para el frenado del complejo.

El 23 de marzo fueron ejecutadas brillantemente las operaciones finales de retirada de la órbita, frenado en la atmósfera y hundimiento del complejo orbital «Mir» en una región segura del océano Pacífico. Ciertamente, este día fue emocionalmente duro para todos los especialistas quienes han dedicado 15 años de su vida a los trabajos del complejo «Mir». Pero los conocimientos y la experiencia obtenidos no se han perdido en vano, éstos se emplean plenamente en el proceso de la construcción de la Estación espacial internacional.

Gracias a la explotación exitosa de muchos años de la estación «Mir», toda la comunidad mundial confió en la solidez de la técnica espacial pilotada y los vuelos de tripulaciones internacionales han permitido ampliar considerablemente la colaboración internacional de muchos años y en muchos aspectos. Actualmente, 20 países del mundo ya tienen su propio programa de vuelos pilotados, o bien tratan de tomar parte en su realización en los próximos años. 


\section{Koptiev Yuri Nikoláyevich}

Datos principales de la estación «MIR"

Composición permanente de la estación «Mir»

\begin{tabular}{||l|c|c|}
\hline $\begin{array}{c}\text { Denominación del elemento de } \\
\text { la estación con la estación }\end{array}$ & Fecha del lanzamiento & Fecha de empalme \\
\hline Bloque básico & 20.02 .1986 & - \\
\hline Módulo «Kvant» & 31.03 .1987 & 09.04 .1987 \\
\hline Módulo «Kvant-2» & 26.11 .1989 & 06.12 .1989 \\
\hline Módulo «Kristall» & 31.05 .1990 & 10.06 .1990 \\
\hline Módulo «Spektr» & 20.05 .1995 & 01.06 .1995 \\
\hline Compartimiento de empalme & 12.11 .1995 & 15.11 .1995 \\
\hline Módulo «Priroda» & 23.04 .1996 & 26.04 .1996 \\
\hline
\end{tabular}

Características principales de la estación "Mir" en su configuración total

\begin{tabular}{||l|c|c|c|c||}
\hline $\begin{array}{c}\text { Denominación del } \\
\text { elemento de } \\
\text { la estación }\end{array}$ & $\begin{array}{c}\text { Masa } \\
\text { Inicial, } \\
\mathrm{t}\end{array}$ & $\begin{array}{c}\text { Longitud } \\
\text { de cada cuerpo, } \\
\mathrm{m}\end{array}$ & $\begin{array}{c}\text { Amplitud de las } \\
\text { baterías solares, } \\
\mathrm{m}\end{array}$ & $\begin{array}{c}\text { Diámetro } \\
\text { máximo, } \\
\mathrm{m}\end{array}$ \\
\hline Bloque básico & 20,9 & 13,13 & 29,7 & 4,15 \\
\hline Módulo «Kvant» & $11,05^{1)}$ & 5,8 & - & 4,15 \\
\hline Módulo «Kvant-2» & 19,5 & 12,4 & 27,35 & 4,35 \\
\hline Módulo «Kristall» & 19,5 & 11,9 & $36^{2)}$ & 4,35 \\
\hline Módulo «Spektr» & 19,34 & 14,44 & 23,3 & 4,1 \\
\hline Compartimiento de empalme & 3,9 & 4,7 & - & 2,2 \\
\hline Módulo «Priroda» & 19,34 & 11,55 & - & 4,1 \\
\hline Nave «Soyuz TM» & 7,07 & 6,98 & 10,6 & 2,72 \\
\hline Nave «Progress M1» & 7,3 & 7,23 & 10,6 & 2,72 \\
\hline
\end{tabular}

\section{Notas:}

1 Con el bloque de servicio - 20,6 t

2 Con dos baterías solares desplegadas múltiples. 
Masa total de la estación (en la configuración completa -con dos naves empalmadas)- más de 140 t.

Masa de los aparatos científicos: aproximadamente 11,5 t.

En la construcción de los aparatos científicos para la estación «Mir» participaron 27 países.

\section{Dimensiones lineales:}

Por cuerpos del bloque principal del módulo «Kvant» y de dos naves empalmadas (por el eje X): aprox. $33 \mathrm{~m}$; 29 m;

Por cuerpos de los módulos «Kvant-2» y «Spektr» (por el eje Y): aprox.

Por cuerpos de los módulos «Priroda» y «Cristall» y del compartimiento del empalme (por el eje Z): aprox. 30 m.

\section{Permanencias en la estación}

Leonid Kizim y Vladímir Soloviov fueron los primeros miembros e la tripulación en la estación «Mir» (tripulación de la primera expedición principal: EO-1). Ellos despegaron en la nave «Soyuz T-15» el 13 de marzo de 1986, y el 15 de marzo llegaron a la estación. En el transcurso de su trabajo esta tripulación realizó el primer traslado interorbital a la estación «Saliut-7», donde llevaron a cabo experimentos científicos, que incluían dos salidas al espacio abierto. Posteriormente, los cosmonautas regresaron a la estación "Mir» llevando consigo cerca de 300 kilos de aparatos científicos.

Durante todo el período de vuelo en la estación «Mir» estuvieron 104 personas.

De éstos:

2 veces -18 personas;

3 veces -4 personas (Serguey Avdéyev, Victor Afanasiev, Aleksandr

Kaleri y el astronauta de USA Charles Prekort);

4 veces -1 persona (Aleksandr Viktorenko);

5 veces -1 persona (Anatoli Soloviov).

En la estación "Mir» estuvieron 62 ciudadanos extranjeros, representantes de 11 países y de la Agencia Espacial Europea (AEE)

Entre ellos:

USA - 44 personas;

Francia - 5 personas;

AEE - 3 personas;

Alemania - 2 personas;

Siria, Bulgaria, Afganistán, Japón, Gran Bretaña, Austria, Canadá,

Eslovaquia - 1 hombre por cada país. 
En la estación «Mir» se realizaron 28 principales expediciones prolongadas, en cuyas tripulaciones trabajaron:

35 cosmonautas de Rusia;

7 astronautas de USA;

1 astronauta de AEE (un ciudadano de Alemania);

1 astronauta de Francia.

Desde el 24 de marzo de 1996 al 8 de junio de 1998, los astronautas de USA permanecieron constantemente en la estación "Mir». El tiempo total de su trabajo como personal de las tripulaciones de las principales expediciones ha sido de 942 días 06 horas 15 minutos.

En la estación «Mir», conjuntamente con las expediciones principales trabajaron 16 expediciones con una duración de permanencia desde una semana hasta un mes. De éstas, 15 eran expediciones internacionales con la participación de Siria, Bulgaria, Afganistán, Francia (5 expediciones), Japón, Gran Bretaña, Austria, Alemania (2 expediciones), AEE y Eslovaquia.

Además, se realizaron nueve expediciones de visita con ayuda de las naves del sistema «Space Shattle», durante los cuales en la estación estuvieron 37 astronautas de USA (además, 6 astronautas se quedaron para un trabajo prolongado entre el personal de las tripulaciones de las expediciones principales), 1 astronauta de Canadá, 1 de AEE, 1 de Francia, y 4 cosmonautas de Rusia.

\section{Vuelos espaciales del hombre más prolongados}

En el transcurso de la explotación de la estación «Mir», se han establecido record mundiales absolutos de tiempo de permanencia continua del hombre en condiciones del vuelo espacial:

1987 - Yuri Romanenko (326 días 11 horas 38 minutos);

1988 - Vladímir Titov, Musa Manarov (365 días 22 horas 39 min.); 1995 - Valeri Poliakov (437 días 17 horas 58 minutos).

En 1995, Valeri Poliakov se convirtió también en recordman absoluto mundial de tiempo total de permanencia en el espacio, en 1999 su éxito lo superó Serguey Avdeyev:

Valeri Poliakov - 678 días 16 horas 33 minutos (en 2 vuelos);

Serguey Avdeyev - 747 días 14 horas 12 minutos (en _ vuelos).

Entre las mujeres los record mundiales de tiempo de vuelo espacial lo establecieron:

1995 - Yelena Kondakova (169 días 05 horas 1 minuto);

1996 - Shannon Lucid, USA (188 días 04 horas 00 min., incluyendo en la estación «Mir» - 183 días 23 horas 00 min.). 


\section{El hombre en el espacio cósmico}

De entre los ciudadanos extranjeros los vuelos más prolongados lo realizaron, según el programa «Mir»:

Jean-Pierre Eniere (Francia)- 188 días 20 horas 16 min.

Shannon Lucid (USA)- 188 días 04 horas $00 \mathrm{~min}$.

Tomas Raiter (AEE, Alemania)- 179 días 01 horas $42 \mathrm{~min}$.

\section{Salidas al espacio abierto}

En la EO (estación orbital) «Mir» se llevaron a cabo 75 salidas al espacio abierto y 3 salidas al módulo deshermetizado "Spektr» con una duración total de 359 horas 12 minutos.

En las salidas participaron:

29 cosmonautas de Rusia;

3 astronautas de USA;

2 astronautas de Francia;

1 astronauta de AEE (ciudadano de Alemania).

Cosmonautas que realizaron seis o más salidas al espacio abierto desde la estación "Mir»

\begin{tabular}{||l|c|c||}
\hline Cosmonauta & Cantidad de módulos & Duración total \\
\hline Anatoli Soloviov & 16 & 77 horas $46 \mathrm{~min}$. \\
\hline Serguey Avdeyev & 10 & 41 horas 59 min. \\
\hline Aleksandr Serebrov & 10 & 31 horas $48 \mathrm{~min}$. \\
\hline Nikolay Budarin & 8 & 44 horas 00 min. \\
\hline Talgat Musabáyev & 7 & 41 horas $18 \mathrm{~min}$. \\
\hline Victor Afanasiev & 7 & 38 horas $33 \mathrm{~min}$. \\
\hline Serguey Krikaliov & 7 & 36 horas $29 \mathrm{~min}$. \\
\hline Musa Manarov & 7 & 34 horas $32 \mathrm{~min}$. \\
\hline Anatoli Artsebarski & 6 & - \\
\hline Yuri Onufriyenko & 6 & 32 horas $17 \mathrm{~min}$. \\
\hline Yuri Usachov & 6 & 30 horas $30 \mathrm{~min}$. \\
\hline Guennadi Strekalov & 6 & 30 horas $30 \mathrm{~min}$. \\
\hline Aleksandr Viktorenko & 6 & 19 horas $39 \mathrm{~min}$. \\
\hline Vasili Tsibliyev & 6 & 19 horas $11 \mathrm{~min}$. \\
\hline \hline
\end{tabular}




\section{Koptiev Yuri Nikoláyevich}

\section{Vuelos de aparatos espaciales hacia la estación «Mir»}

Hacia la estación «Mir» realizaron vuelos y empalmes $101 \mathrm{AE}$ (aparatos espaciales) de Rusia: «Soyuz-T», «Soyuz-TM», «Progress»; los módulos: «Kvant», «Kristall», «Spektr», «Priroda».

Además de esto, hacia la estación «Mir» se realizaron 10 vuelos y se llevaron a cabo 9 empalmes de las naves americanas del sistema «Space Shattle»: 7 vuelos fueron del «Atlantic», 2 - «Discovery» (en el primer vuelo del «Discovery» no se había previsto el empalme), 1 - «Indevor». 